\title{
Antithrombotics from Frog Skin Secretions
}

\author{
Heyu $\mathrm{Ni}^{1,2,3,4,5,6}$ \\ ${ }^{1}$ Department of Laboratory Medicine, Keenan Research Centre for \\ Biomedical Science-Li Ka Shing Knowledge Institute, St. Michael's \\ Hospital, Toronto, Ontario, Canada \\ 2 Toronto Platelet Immunobiology Group, Toronto, Ontario, Canada \\ ${ }^{3}$ Canadian Blood Services Centre for Innovation, Toronto, Ontario, \\ Canada \\ ${ }^{4}$ Department of Laboratory Medicine and Pathobiology, University of \\ Toronto, Toronto, Ontario, Canada \\ ${ }^{5}$ Department of Physiology, University of Toronto, Toronto, Ontario, \\ Canada \\ ${ }^{6}$ Department of Medicine, University of Toronto, Toronto, Ontario, \\ Canada
}

Thromb Haemost 2020;120:1351.

In this issue of Thrombosis and Haemostasis, Shen et al described a non-RGD 15-mer peptide isolated from skin secretions of the frog Amolops loloensis, which could selectively bind to activated platelet $\alpha \operatorname{IIb} \beta 3$ integrin and inhibit platelet aggregation induced either by physiological agonists or by an allosteric activator targeting the $\alpha$ IIb transmembrane domain (anti- $\alpha$ IIb TM). This peptide, named Zongdian platelet inhibitor (ZDPI) was able to reduce binding of activated platelets to soluble fibrinogen, and to suppress platelet spreading (but not adhesion) on immobilized fibrinogen. Importantly, ZDPI could inhibit carotid arterial thrombosis without inducing significant bleeding or thrombocytopenia in mice, making it a promising candidate or template to develop antithrombotics with reduced side effects. ${ }^{1}$

It is interesting that ZDPI (FRGCWLKNYSPRGCL-NH $\mathrm{N}_{2}$ ) neither contains a RGD motif nor the key negatively charged "D," which has been considered important for integrin recognition. ${ }^{2}$ Consistently, RGD peptides could not inhibit its binding to $\alpha \mathrm{IIb} \beta 3$. The authors proposed it could bind to the fibrinogen and fibronectin recognition area on $\alpha \operatorname{IIb} \beta 3$ since it interfered with binding from these integrin ligands to platelets. Alternatively, it may bind to an area that regulates integrin conformation, such as the integrin PSI domain. We indeed recently demonstrated that integrin family PSI domains exerted endogenous thiol isomerase function and that targeting these could inhibit $\alpha$ IIb $\beta 3$-ligand interactions and thrombosis. ${ }^{3}$ Since ZDPI has two cysteine residues, it would be interesting to explore whether disulfide bond exchange between ZDPI and PSI domain or other cysteinerich domains of $\alpha \mathrm{IIb} \beta 3$ can occur, and whether this could subsequently downregulate $\alpha \operatorname{Ilb} \beta 3$ affinity for its ligands.

Address for correspondence Heyu Ni, MD, PhD, Department of Laboratory Medicine, Keenan Research Centre for Biomedical Science, St. Michael's Hospital, Room 421, LKSKI-Keenan Research Centre, 209 Victoria Street, Toronto, Ontario M5B 1W8, Canada

(e-mail: heyu.ni@unityhealth.to; chenp@smh.ca).

Although a promising antithrombotic candidate, ZDPI has a relatively short half-live in vivo and its efficacy is weaker than Integrilin, calling for further modifications toward drug development.

\section{Funding}

This study was funded by Canadian Institutes for Health Research (foundation grant: 389035) and Heart and Stroke Foundation of Canada (grant: G-17-0018663).

\section{Conflict of Interest}

H.N. does not think it impacted on his evaluation and commentary for this paper, but one of the co-first authors has recently joined his lab as a postdoctoral fellow. He informed the associate editor for the situation and asked an additional reviewer to evaluate the manuscript during the manuscript evaluation process. He does think this is a very interesting paper, and somewhat an unexpected discovery, which is deserved to have an editorial commentary "T\&H Insights" as he wrote.

\section{References}

1 Shen C, Liu M, Tian H, et al. Conformation-specific blockade of $\alpha \operatorname{IIb} \beta 3$ by a non-RGD peptide to inhibit platelet activation. Thromb Haemost 2020;120(10):1432-1441

$2 \mathrm{Ni} \mathrm{H}$, Freedman J. Platelets in hemostasis and thrombosis: role of integrins and their ligands. Transfus Apheresis Sci 2003;28(03): 257-264

3 Zhu G, Zhang Q, Reddy EC, et al. The integrin PSI domain has an endogenous thiol isomerase function and is a novel target for antiplatelet therapy. Blood 2017;129(13):1840-1854

(c) 2020 Georg Thieme Verlag KG Stuttgart · New York
DOI https://doi.org/ $10.1055 / \mathrm{s}-0040-1715898$. ISSN 0340-6245. 